\title{
Location cuing benefits with exposure duration variations
}

\author{
A. H. C. VAN DER HEIJDEN, M. NEERINCX, and G. WOLTERS \\ University of Leiden, Leiden, The Netherlands \\ (Charles W. Eriksen, Sponsor)
}

\begin{abstract}
In studies that clearly show that location cuing benefits in single-item recognition tasks are a linear function of the room for improvement, researchers have varied room for improvement by manipulating the distance of targets to the fixation point. To show that such benefits are not the result of probing different parts of the retina, we manipulated room for improvement in this study by varying the presentation time of targets. The same relationship between location cuing benefits and room for improvement was found. Therefore, it is parsimonious to conclude that it is indeed room for improvement, and not retinal position or exposure duration, that determines the size of the cuing effect. A tentative model for explaining location cuing benefits is presented. The model shows that an uninterpretable empirical relationship can be rewritten into a theoretically interpretable form.
\end{abstract}

The first attempts to provide evidence of location cuing benefits in single-item recognition tasks with accuracy as the dependent variable were failures (see, e.g., Grindley \& Townsend, 1968). Recently, however, it has been shown that reliable effects can be found (Egly \& Homa, 1984; Van der Heijden, Schreuder, \& Wolters, 1985; Van der Heijden, Wolters, Groep, \& Hagenaar, 1987).

Van der Heijden et al. (1987) argued that it is easier to demonstrate benefits in tasks using latency than in tasks using accuracy as the dependent variable. In latency tasks, improvements by cuing are in principle possible in all trials. In accuracy tasks, however, there is already a proportion correct without cuing $\left(I_{\mathrm{nc}}\right)$. Thus, there is room for improvement only on a proportion of trials $\left(1-I_{\mathrm{nc}}\right)$. Van der Heijden et al. supported this view by showing that in all relevant experiments, the improvement in recognition accuracy by cuing $(\Delta X)$ can be expressed as a linear function of $\left(1-I_{\mathrm{nc}}\right)$ :

$$
\Delta X=a\left(1-I_{\mathrm{nc}}\right)+b, \quad a>0 .
$$

The studies that systematically varied $\left(1-I_{\mathrm{nc}}\right)$, however, have left one important question open. Both Egly and Homa (1984) and Van der Heijden et al. (1987) varied $\left(1-I_{\mathrm{nc}}\right)$ by manipulating the distance of the targets to the fixation point. By varying this distance, however, parts of the retina are probed that differ in resolution power. Therefore, the results are ambiguous with regard to the question of whether it is $\left(1-I_{\mathrm{nc}}\right)$ or retinal position that determines the size of the cuing effect. To dissolve this ambiguity, it is worthwhile to investigate whether the relationship between $\Delta X$ and $\left(1-I_{\text {nc }}\right)$ also holds when accuracy is manipulated by varying the presentation time

Address correspondence to A. H. C. van der Heijden, Department of Psychology, Unit of Experimental Psychology, University of Leiden, Hooigracht 15, 2312 KM Leiden, The Netherlands. of targets and keeping the distance of targets to the fixation point constant. If the same result is found, it is parsimonious to conclude that it is $\left(1-I_{\mathrm{nc}}\right)$, not retinal position or exposure duration, that determines the size of the cuing effect.

In the experiment reported here, only one distance between targets and fixation point was used. A large range of $\left(1-I_{\mathrm{nc}}\right) \mathrm{s}$ was induced by varying the exposure duration.

\section{METHOD}

\section{Subjects}

Eight students of the University of Leiden served as paid subjects. All had normal or corrected-to-normal vision.

\section{Apparatus}

The stimuli were presented on a display screen (VR-17). Stimulus presentation and response registration were controlled by a DEC PDP$11 / 40$ computer. The subjects initiated stimulus exposure by pressing a button in the table surface in front of them.

\section{Stimuli}

Each stimulus consisted of one capital letter, chosen from a set of eight ( $\mathrm{E}, \mathrm{F}, \mathrm{H}, \mathrm{M}, \mathrm{K}, \mathrm{X}, \mathrm{O}$, and $\mathrm{Q})$. A letter subtended a visual angle of $.31^{\circ} \times .42^{\circ}$ at a viewing distance of $55 \mathrm{~cm}$. A letter was presented in a position chosen from five equally spaced positions on the circumference of an imaginary circle (radius $2.3^{\circ}$ ) around the fixation point (a dim asterisk). One of the positions was at 12:00.

As a cue, a low-intensity dot was presented at the position of the impending target. In the no-cue condition, dots were presented simultaneously on all five possible target locations. ${ }^{1}$

\section{Design}

A within-subject design with repeated measures was employed. Five different exposure durations were used: $5,25,45,65$, and $85 \mathrm{msec}$. The 400 different presentations were derived from an 8 (letters) $\times 5$ (positions) $\times 2$ (cue, no-cue) $\times 5$ (exposure durations) design. Each subject saw these presentations twice. Four sessions were run, each containing 200 trials. The order of trials was determined randomly, with the restriction that position, letter, cuing condition, and exposure duration were balanced over the first and the second two sessions. 
One session of practice preceded the four experimental sessions. The sessions took place on 5 consecutive days. In the experimental sessions, subjects started with $\mathbf{4 0}$ practice trials.

\section{Procedure}

Each subject was run individually in a dimly illuminated room. The subject was seated at a table and instructed to look at the display until he/she clearly saw the fixation point. After pressing a button in front of him/her, the fixation point disappeared and the following presentation sequence occurred: cue (one dot) or no-cue (five dots) exposure for $25 \mathrm{msec}$; a subsequent delay of $25 \mathrm{msec}$, during which the screen was empty; and exposure of a letter in one of the five locations for 5 , $25,45,65$, or $85 \mathrm{msec}$. The subject was required to respond by naming the letter.

\section{RESULTS AND DISCUSSION}

Correct letter identification scores were determined for each subject, condition (cue and no-cue), and exposure duration. Each score was based on 80 trials. An analysis of variance showed that the main effects of cuing $[F(1,7)=13.980, p<.01]$ and of exposure duration $[F(4,28)=91.708, p<.001]$ were highly significant. Table 1 presents the relevant results for the five exposure durations, together with the values of room for improvement $\left(1-I_{\mathrm{nc}}\right)$ and benefits $(\Delta X)$. The table shows that position cuing enhances identification performance. It also suggests that this enhancement increases as room for improvement increases. To further investigate whether $\Delta X$ linearly increases with $\left(1-I_{\mathrm{nc}}\right)$, a linear function (Equation 1) was fitted.

Table 2 presents the correlations and parameters overall and separately per exposure duration. The correlations are lower than in earlier studies; however, in this study, the correlations were determined with values averaged over 10 observations. This introduced a large error variation, which reduces the correlations but enhances the degrees of freedom. Although the correlation for the total data set appears to be quite low, it is highly significant $(p<.01$ for $r>.144)$. Moreover, the fit of the linear function was better than the fit of any of a number of nonlinear functions.

In sum, the results of this study are in accordance with previous experiments demonstrating cuing effects in a single-item recognition task with accuracy as the dependent variable (Van der Heijden et al., 1985; Van der Heijden et al., 1987). Furthermore, it appears that when room

Table 1

Proportions Correct per Exposure Duration (in msec) and Cuing Condition, the Corresponding Rooms for Improvement $\left(1-I_{n c}\right)$, and Benefits $(\Delta X)$

\begin{tabular}{|c|c|c|c|c|}
\hline \multirow{2}{*}{$\begin{array}{l}\text { Exposure } \\
\text { Duration }\end{array}$} & \multicolumn{2}{|c|}{ Condition } & \multirow[b]{2}{*}{$\left(1-I_{\mathrm{nc}}\right)$} & \multirow[b]{2}{*}{$\Delta X$} \\
\hline & Cue & No Cue & & \\
\hline 5 & .241 & .186 & .814 & .055 \\
\hline 25 & .402 & .364 & .636 & .038 \\
\hline 45 & .555 & .519 & .481 & .036 \\
\hline 65 & .591 & .567 & .433 & .024 \\
\hline 85 & .631 & .598 & .402 & .033 \\
\hline$M$ & .484 & .447 & .553 & .037 \\
\hline
\end{tabular}

Table 2

Correlations $(R)$ and Parameters $(a, b)$ for the Linear Functions Given by Equation 1 per Exposure Duration (in milliseconds) and Over the Total Data Set (AII)

\begin{tabular}{cccc}
\hline $\begin{array}{c}\text { Exposure } \\
\text { Duration }\end{array}$ & $R$ & $a$ & $b$ \\
\hline 5 & .222 & .187 & -.097 \\
25 & .341 & .252 & -.123 \\
45 & .191 & .139 & -.031 \\
65 & .258 & .146 & -.040 \\
85 & .166 & .098 & -.007 \\
All & .232 & .138 & -.039 \\
\hline
\end{tabular}

for improvement is varied by manipulating exposure duration instead of distance of the targets from the fixation point, benefits brought by cuing are also a function of $\left(1-I_{n c}\right)$. Thus, it is parsimonious to conclude that it is $\left(1-I_{n c}\right)$, and not retinal position or exposure duration, that determines the size of the cuing effect.

\section{A TENTATIVE MODEL}

As a model for benefits of cuing, Equation 1 has the serious drawback of being very difficult to interpret. A rewriting of this equation makes this readily apparent:

$$
\Delta X=a\left\{[1+(b / a)]-I_{\mathrm{nc}}\right\} \text {. }
$$

In this form, it is clear that $\Delta X=0$, not for $I_{\mathrm{nc}}=1$ as one would expect, but for $I_{\mathrm{nc}}=1+(b / a)$. If actual values from our tables are used, it appears that $1+(b / a)<1$. However, then Equation 1 or Equation 2 as a model suggests that for values of $I_{\mathrm{nc}}>1+(b / a)$, the cuing effect is negative! Moreover, Equation 2 states that for all exposure durations for which $I_{\mathrm{nc}}$ is at chance level, maximal cuing benefits are to be expected. Both predictions are intuitively unlikely and not apparent in the data. Thus, we have to look for an alternative for Equation 2 that removes this theoretical inconsistency.

A suggestion for an alternative can be derived from evidence reported by Egly and Homa (1984), whose study involved one letter naming task and two position naming tasks. The letter naming task was not too different from the task reported here. In the position naming task, subjects had to name only the position of the letter. The remarkable observation was that in the position naming task, with exposure durations rather similar to those used in the letter naming task, quite a lot of errors were made. Moreover, benefits brought by cuing were larger for position than for identity information. Also, in one of our studies (Van der Heijden, Fleur, \& Wolters, 1988) we found that without cuing, subjects could not report the position of the target in a significant number of cases, and that position enhancement by cuing was larger than identity enhancement. Thus, it appears that in these types of tasks, subjects quite often have no position information and that cuing especially improves position information. Therefore, it is possible that position information, and not identity information, is at the basis of the cuing effect, 
or, that cuing may be effective because it disambiguates position uncertainty and not because it enhances identity information.

One definite possibility, then, is that benefits brought by cuing are a fraction of the improvement, not in terms of identity information, but in terms of position information, that is, that

$$
\Delta X=g\left(P_{\mathrm{c}}-P_{\mathrm{nc}}\right) .
$$

In this equation, $P_{\mathrm{c}}$ is the proportion of trials in which position information is available in the cue condition, and $P_{\mathrm{nc}}$ is the proportion of trials in which position information is available in the no-cue condition. If we assume that cuing in our task results in perfect position informationthat is, that $P_{\mathrm{c}}=1-$ Equation 3 becomes

$$
\Delta X=g\left(1-P_{\mathrm{nc}}\right) \text {. }
$$

(Remember that the cue was clearly visible and reliably indicated the position of the impending target.)

It is clear that Equation 3 does not suffer from the drawbacks of Equations 1 and 2. If $P_{\mathrm{nc}}=1$, its maximum value, then $\Delta X=0$. This makes sense: no further increase in identification performance by spatial cuing is to be expected if there is already perfect localization performance in the no-cue condition. If $\boldsymbol{P}_{\mathrm{c}}$ is at chance level, then $\boldsymbol{P}_{\mathrm{nc}}$ also will necessarily be at chance level and no benefits are to be expected.

It is essential to note that Equation 4-that is, Equation 3 with the additional assumption that cuing in our task results in perfect position information-is consistent with Equations 1 and 2 . If we assume that in the no-cue condition only a fraction $(s<1)$ of the localized items are correctly recognized, that is, that

$$
I_{\mathrm{nc}}=s P_{\mathrm{nc}}, \quad s<1,
$$

and therefore that

$$
(1 / s) I_{\mathrm{nc}}=P_{\mathrm{nc}},
$$

Equation 4 can be rewritten as

$$
\Delta X=g\left[1-(1 / s) I_{\mathrm{nc}}\right] .
$$

Because

$$
\Delta X=g-\left[g(1 / s) I_{\mathrm{nc}}\right]+[g(1 / s)]-[g(1 / s)],
$$

it follows that

$$
\Delta X=\left[g(1 / s)\left(1-I_{\mathrm{nc}}\right)\right]+g-[g(1 / s)] .
$$

Thus, Equation 6 is of the form

$$
\Delta X=a\left(1-I_{\mathrm{nc}}\right)+b,
$$

with $a=g(1 / s)$ and $b=g[1-(1 / s)]$.

Fortunately, it is possible to check the correctness of this analysis. Equation 1 (with the observable $I_{\text {nc }} s$ ) provides a means to test Equation 4 (with the nonobservable $P_{\text {nc }}$ ) as a model, because a number of interesting theoretical predictions can be verified. We mention some of these predictions and investigate their correctness by ex- amining the parameters obtained for the different exposure durations (i.e., the parameters in Table 2).

First, from Equations 1 and 6, it follows that

$$
a+b=g(1 / s)+g[1-(1 / s)]=g .
$$

Because in Equation $4 \mathrm{~g}$ stands for a positive fraction (i.e., $0<g<1$ ) the sums of the parameters for each function in Table 2 also have to be positive fractions. The values obtained indeed appear to be positive fractions (see Table 3).

Second, with $g$ given and with the knowledge that $a=g(1 / s)$ or $b=g[1-(1 / s)]$, it is easy to find $1 / s$ and $s$. Because $I_{\mathrm{nc}}=s P_{\mathrm{nc}}$ (i.e., the proportion correctly identified is a fraction, $s$, of the proportion correctly localized), all $s$ have to be smaller than 1 (and all 1/s larger than 1) for the model to be correct. These values are indeed positive fractions (see Table 3).

Third, with $1 / s$ given and with $I_{\mathrm{nc}}$ as data, it is easy to obtain estimates of the not directly observable localization performance $\left(P_{\mathrm{nc}}\right)$ by means of Equation 5 . Because $P_{n c}$ stands for the proportion of trials with correct localization of the letter, the values obtained have to be equal to or smaller than 1 (i.e., $P_{\mathrm{nc}} \leq 1$ ). Again, the estimates are in accordance with this prediction (see Table 3).

The three predictions and the data in Table 3 show that the model is at least qualitatively consistent with the data. It therefore seems that we are on the way from an uninterpretable empirical relation (Equation 1) to an interpretable model (Equation 4). However, quantitatively, there remains much to be desired. A number of reasons can be advanced to explain the quantitative discrepancies.

First, in the type of task under discussion, it is very likely that guessing is involved. It is far from clear how guessing affects performance and especially how it affects the parameters estimated with Equation 1.

Second, for estimating the parameters, $\Delta X$ was determined as a function of $\left(1-I_{\mathrm{nc}}\right)$. Theoretically, however, there is no reason to prefer this relation over $\left(1-I_{\mathrm{nc}}\right)$ as a function of $\Delta X$. The two regression equations lead to different estimates of the parameters. At present, it is not known what the best way to obtain the parameters is.

Third, care has to be taken in interpreting the parameters. Given the evidence of the present identification task only, we do not really know what $g$ and $s$ stand

Table 3

Estimates of the Theoretical Parameters $g, s$, and $\boldsymbol{P}_{\mathrm{nc}}$ per Exposure Duration (in milliseconds) and Overall

\begin{tabular}{cccc}
\hline $\begin{array}{c}\text { Exposure } \\
\text { Duration }\end{array}$ & $g$ & $s$ & $\boldsymbol{P}_{\text {nc }}$ \\
\hline 5 & .090 & .481 & .387 \\
25 & .129 & .513 & .710 \\
45 & .108 & .775 & .670 \\
65 & .106 & .725 & .782 \\
85 & .091 & .926 & .646 \\
Overall & .099 & .717 & .623 \\
\hline
\end{tabular}

Note-See text for further explanation. 
for. Moreover, we do not know whether the assumption of perfect localization performance in the cuing condition is really correct (i.e., whether Equation 4 or Equation 3 is the correct model). Further research using localization and identification tasks is needed to arrive at the correct interpretation of the parameters.

\section{REFERENCES}

Egly, R., \& НомA, D. (1984). Sensitization of the visual field. Journal of Experimental Psychology: Human Perception \& Performance, 10, 778-793.

Grindley, G. C., \& Townsend, V. (1968). Voluntary attention in peripheral vision and its effects on acuity and differential thresholds. Quarterly Journal of Experimental Psychology, 20, 11-19.

Van der Heijden, A. H. C., Fleur, E., \& Wolters, G. (1988). The effect of cueing on location and identification of single items (Internal Report). Leiden, The Netherlands: Leiden University, Department of Psychology.

Van der Heuden, A. H. C., Schreuder, R., \& Wolters, G. (1985). Enhancing single-item recognition accuracy by cueing spatial locations in vision. Quarterly Journal of Experimental Psychology, 37A, 427-434.
Van der Heijden, A. H. C., Wolters, G., Groep, J. C., \& HAGENAAR, R. (1987). Single-letter recognition accuracy benefits from advance cuing of location. Perception \& Psychophysics, 42, 503-509.

\section{NOTE}

1. A condition in which the cue and no-cue dots were presented slightly further away from the fixation point than the target letters $\left(2.7^{\circ} \mathrm{vs} .2 .3^{\circ}\right.$ of visual angle) did not produce a cuing benefit (overall proportions correct in the cue and no-cue conditions were 0.462 and 0.460 , respectively). However, in this condition, all subjects reported a clear apparent movement phenomenon from cue position to target position Therefore, subjects were able, in the cue condition, as well as in the no-cue condition, to determine the location of the target correctly in all trials. As a result, the beneficial effect of cuing (i.e., providing unambiguous location information) over no-cuing disappeared. This explanation is completely in accordance with the model presented later in this paper, which suggests that there is no further increase in identification performance given perfect localization performance. In fact, it was this observation that led us to the formulation of the model presented. 